\title{
Editorial
}

\section{LOW PERMEABILITY MEDIA: A RESEARCH FRONTIER}

\author{
Henri Van Damme \\ Member of Scientific board of IFP Energies nouvelles \\ ESPCI-PCSM, 10, rue Vauquelin, 75231 Paris cedex 05
}

This thematic dossier collects eight scientific papers corresponding to presentations made during two international conferences dedicated to the study of low permeability media held in Paris within six months of each other. The first conference, "The Challenge of Studying Low Permeability Materials: Laboratory, in situ (field) and numerical methods"1, was organised in December 2014 by the University of Cergy-Pontoise (Geosciences and Environment Cergy Research Laboratory) under the aegis of CFMR (Rock Mechanics French Committee) and GIS Géosciences franciliennes ${ }^{2}$. The second, LowPerm $2015^{3}$ "Low permeability media and nanoporous materials from characterisation to modeling: Can we do it better?", was organised by IFP Energies nouvelles in June 2015 under the aegis of the French "Académie des Sciences".

An attractive and explicit title is key to the success of a conference. It sets the scene and lets possible participants know exactly what matter will be discussed. But subtitles may be as important as titles, because they may stimulate participants by confronting them with a collective challenge. Devoted to characterisation, transport properties and multiscale modeling of low to very low permeability media and nanoporous materials, LowPerm 2015 was leaving very little ambiguity about the matter to be discussed. Its subtitle - Can we do better? - was a mild way of stressing that there are several areas where we should be totally unsatisfied about our present state of knowledge, and where progress is urgently needed.

One of these areas is the recovery of unconventional hydrocarbon resources. There is no question that the gas and oil shale revolution has deeply changed the global energy landscape, but its long term sustainability is questionable in spite of the tremendous magnitude of the resource due to an unacceptably low rate of recovery. With close to seventy five percent of the gas and ninety five percent of the oil left in place on average, the exploitation of self-sourced organic rich shale reservoirs is one of the least efficient industrial operations. Among the main reasons for this are our poor knowledge of the complex nanoporous void space in which the hydrocarbons are trapped, and our very limited understanding of their release and transport mechanisms, particularly in multiphase conditions. Just imagine what the future would be if, thanks to improved understanding, we could switch from ninety five percent left in place to ninety five percent recovery!

\footnotetext{
${ }^{1}$ This conference was organised by Christian David and Jérôme Wassermann.

${ }^{2}$ The GIS Géosciences franciliennes is a scientific collaboration between ten public institutions from Ile-de-France. It aims to pool research efforts on issues related to natural resources and environment which are the subject of major societal concerns: www.geosciences-franciliennes.fr.

${ }^{3}$ LowPerm 2015 was organised by Noalwenn Dubos-Sallée. The program and some of the presentations can be downloaded at: www.rs-lowperm2015.com.
}

This is an Open Access article distributed under the terms of the Creative Commons Attribution License (http://creativecommons.org/licenses/by/4.0), which permits unrestricted use, distribution, and reproduction in any medium, provided the original work is properly cited. 
Nuclear waste storage is another area - not so far from the previous one - where progress is needed. Several countries, including France, are considering storing their radioactive wastes in deep low permeability clay-containing geological formations. Just like organic rich shales, these formations are highly heterogeneous. Furthermore, their nano- and meso-structure is sensitive to the thermal, hydric, mechanical and chemical gradients induced by the stored wastes. Modeling their very long term behaviour is a formidable challenge, at the limit of what is reasonably possible. The same could be said about the cap rocks that would guarantee the perennial storage of carbon dioxide if this turns out to be an economically viable carbon mitigation strategy.

As far as material science and chemical engineering are concerned, there are many examples where a better understanding of low permeability physics would be useful. One might think of heterogeneous catalysts, packaging materials or desalination polymer membranes, just to name a few. Being the most widely used man made material, concrete or, more generally, cement-based materials, are also low permeability media where progress is sorely needed. Obtained by firing clay and limestone at high temperature, cement is best considered as an artificial igneous rock. Its reaction with water is a kind of accelerated weathering process leading to a dense mixture of hydrated minerals - the so-called cement hydrates - akin to a sedimentary rock. Hardened cementitious materials are generally very low permeability materials. Yet permeability is a key property, because it largely controls their durability in wet environments.

Broadly speaking, the questions raised by low permeability materials fall into two categories. One category gathers the questions related to very small pore sizes, whereas the other is related to the complex and often hierarchical or multiscale character of the void space. Among the questions falling into the first category, one may mention the influence of pore size, pore wall wettability, pore wall curvature, or pore boundaries. This physics does not require complex systems to be investigated. It can be studied in simple model systems like nanotubes, molecular sieves or porous silicon, either through real experiments or by molecular simulation. One simple and yet very relevant question - in particular for shales or polymers - is the nature of molecular transport in the extreme case of sub-nanoporous materials, where the pore size is of the same order of magnitude as the molecular diameter, i.e. in conditions where the basic concepts of hydrodynamics no longer apply. What recent studies have shown is that flow in these conditions looks very much like total slip with friction. This is akin to reptation, the type of diffusive motion encountered in polymer melts, where each polymer molecule is sliding in its "tube". Interestingly, in the case of a single fluid, this does not challenge the linear relationship between flow rate and pressure gradient (or more generally, between flow rate and chemical potential gradient). But it modifies the physical meaning of the proportionality coefficient (the permeance), which is no longer the ratio of a material property (permeability) and a fluid property (viscosity).

As a matter of fact, slip - possibly with friction - remains an important issue in a wide range of conditions, including in electrokinetic flows. In general, the Knudsen number remains the primary parameter to consider for estimating the possible need to introduce slip boundary conditions, but it appears that the Klinkenberg treatment of slip is no longer sufficient. The absolute value of the pore size and the curvature of the pore wall seem to be important additional parameters to consider. How this might be related to adsorption phenomena remains to be established.

Another class of phenomena which deserves more investigation is that of interface-controlled barriers. This may for instance happen when a hydrocarbon fluid phase trapped in a nanoporous hydrophobic medium (kerogen) has to escape into a hydrophilic medium (water-filled fractures). In this case, the rate of escape is no longer controlled by the transport properties, but by the interfacial energy barrier for the nucleation and growth of droplets or bubbles at the hydrophobichydrophilic interface.

Much more intriguing is the difference between theoretical and measured transport properties (either diffusivity or permeability) in simple nanoporous model systems like zeolithic or porous silicon membranes. Experimental data seem to be systematically much lower than the values predicted by classical models. Interface barriers at the entrance of the nanopores have been invoked, but a physical explanation for the existence of such barriers is still lacking. 
The involvement of the granular character of molecules at these very small length scales is suspected, but this remains the subject of much conjecture.

When taken together, the various phenomena that were just mentioned - slip, friction, adsorption, wetting, curvature, interface barriers etc. - all of which were discussed during LowPerm 2015, represent a formidable challenge. Whether they are collectively prone to a common treatment remains to be established.

A second and quite different category of questions raised by low permeability media are those related to the complex and often multiscale character of the void space. In the era of big data, many of us are dreaming of the ultimate technique that would allow for the mapping of a Representative Elementary Volume (REV) with atomic resolution. While this might become reality in the nottoo-distant future, it is not necessarily a desirable option. More precisely, even such a fantastic amount of structural information wouldn't deliver all of what we are looking for. For instance, even a simple concept like tortuosity is not of purely geometrical nature. It is critically dependent on the short-time interfacial molecular dynamics. The answers to questions such as "How much time does a molecule spend on the wall of a given pore family before returning to the bulk space?" are as important as the void space topology (connectivity) for estimating transport restriction factors. Rather than expecting the ultimate geometrical information from a single ideally powerful technique, it is probably wiser to operate a number of techniques able to deliver specific information, either in real or in Fourier space. Among the various techniques that one may consider, low field nuclear magnetic resonance deserves a special mention. It provides access to a remarkably wide range of time and length scales and it has a unique capacity to correlate dynamics in different families of pores.

A particularly challenging situation is that of (micro)fractured (sub)nanoporous media, where the (sub)nanopores are the voids where molecules are strongly adsorbed, whereas the (micro)fractures are the main escape paths. This is probably a good model for production of hydrocarbons from organic rich shales. It is a challenging situation not only for characterisation, but also for modeling. Due to the very different length scales and dynamics in the two types of porosity, it is unlikely that classical dual porosity models could work. In addition, a strong dependence of the (micro)fractured network on the state of mechanical stress is expected. The sensitivity to the state of stress could be even higher if the moving molecules are dissolving in the matrix, instead of occupying pre-existing pores. This situation, with concomitant solid volume changes, occurs in polymer barriers, in swelling claystones, and perhaps in kerogen.

Once again, when taken together, the different sources of structural and/or dynamic heterogeneity represent a formidable challenge for upscaling our models to the macroscopic scale. Much like what we saw at a local level, there is little hope that this could be achieved by presupposing a single type of flow (Darcy, diffusive, anomalously diffusive) and decoupling the fluid properties (density, mobility, residence time, etc.) from the solid structure. Using step-wise upscaling methods based on Onsager's and Maxwell-Stefan linear response theories to relate fluxes to chemical potential gradients with a structure-dependent mobility factor, possibly with the help of molecular simulation methods, may well prove to be a more powerful approach. 



\title{
Éditorial
}

\section{MILIEUX PEU PERMÉABLES : UNE FRONTIÈRE POUR LA RECHERCHE}

\author{
Henri Van Damme \\ Membre du conseil scientifique IFP Energies nouvelles \\ ESPCI-PCSM, 10, rue Vauquelin, 75231 Paris cedex 05
}

Ce dossier thématique regroupe huit articles scientifiques qui correspondent aux présentations données lors de deux conférences internationales dédiées à l'étude des milieux peu perméables, qui se sont tenues à Paris à moins de six mois l'une de l'autre. La première "Défi lié à l'étude des matériaux peu perméables : méthodes de laboratoire, in situ (sur le terrain) et numériques $»^{1}$ a été organisée en décembre 2014 par l'Université de Cergy-Pontoise (Laboratoire de recherche en géosciences et environnement de Cergy) sous l'égide du CFMR (Comité Français de Mécanique des Roches) et le GIS Géosciences franciliennes ${ }^{2}$. La deuxième, LowPerm $2015^{3}$ "De la caractérisation à la modélisation des milieux peu perméables et des matériaux nanoporeux : quelles améliorations possibles? » a été organisée par IFP Energies nouvelles en juin 2015 sous l'égide de l'Académie des Sciences.

Un titre attrayant et explicite est la clé du succès d'une conférence. Il plante le décor et permet aux éventuels participants de connaître exactement la thématique dont il sera question. Mais les sous-titres peuvent être tout aussi importants que les titres car ils sont à même de stimuler les participants en les mettant face à un défi collectif. Dédiée à la caractérisation, aux propriétés de transport et à la modélisation multi-échelle des milieux peu à très peu perméables et des matériaux nanoporeux, LowPerm2015 laissait très peu d'ambiguité quant à la thématique dont il serait question. Son sous-titre - Quelles améliorations possibles ? - était une façon complaisante de souligner qu'il existe de nombreux domaines pour lesquels nous devrions considérer l'état actuel de nos connaissances comme entièrement insatisfaisant et où des progrès doivent être accomplis de toute urgence.

L'un de ces domaines est la récupération des ressources en hydrocarbures non conventionnels. Il ne fait aucun doute que la révolution du gaz et du pétrole de schiste a profondément modifié le paysage mondial de l'énergie, mais en dépit des ressources considérables, sa viabilité à long terme est discutable en raison d'un taux de récupération beaucoup trop faible. Avec près de soixantequinze pour cent du gaz et quatre-vingt cinq pour cent du pétrole laissés en place en moyenne, l'exploitation des gisements de schiste riche en matière organique est l'une des activités industrielles dont le rendement est le plus faible. Cela s'explique principalement par notre connaissance insuffisante de l'espace vide nanoporeux complexe dans lequel les hydrocarbures sont piégés, et par notre compréhension très partielle de leurs mécanismes de libération et de transport, en particulier dans des conditions multiphasiques. Imaginez ce que l'avenir serait si, grâce à une

\footnotetext{
${ }^{1}$ Cette conférence a été organisée par Christian David et Jérôme Wassermann.

${ }^{2}$ Le GIS Géosciences franciliennes est un groupement d'intérêt scientifique entre dix établissements publics d'Ile-deFrance. Il vise à fédérer les efforts de recherche sur les problématiques liées aux ressources naturelles et à l'environnement, objets d'une forte demande sociétale. www.geosciences-franciliennes.fr.

${ }^{3}$ La conférence LowPerm 2015 a été organisée par Noalwenn Dubos-Sallée - Le programme et certaines des présentations peuvent être téléchargés à l'adresse www.rs-lowperm2015.com.
} 
meilleure compréhension, nous pouvions passer de quatre-vingt cinq pour cent laissés en place à quatre-vingt cinq pour cent de récupération...

Le stockage des déchets nucléaires est un autre domaine - pas si éloigné du précédent - pour lequel des progrès sont nécessaires. Plusieurs pays, dont la France, envisagent de stocker leurs déchets radioactifs dans des formations géologiques argileuses profondes et peu perméables. Tout comme les schistes riches en matière organique, ces formations sont très hétérogènes. En outre, leur nano- et mésostructure est sensible aux gradients thermique, hydrique, mécanique et chimique induits par les déchets stockés. La modélisation de leur comportement à très long terme constitue un formidable défi, à la limite de ce qui est raisonnablement possible. Le même constat pourrait être fait concernant les roches couvertures qui garantiraient un stockage pérenne du dioxyde de carbone, si cela s'avérait être une stratégie économiquement viable pour la séquestration du carbone.

En termes de science des matériaux et de génie chimique, de nombreux exemples montrent qu'une meilleure compréhension de la physique des milieux peu perméables serait utile. On peut penser aux catalyseurs hétérogènes, aux matériaux d'emballage ou aux membranes polymères de dessalement, pour n'en citer que quelques-uns. S'agissant d'un des matériaux de synthèse les plus couramment utilisés, le béton et, d'une manière plus générale, les matériaux à base de ciment représentent également une classe de matériaux peu perméables pour lesquels des progrès sont attendus. Étant obtenu par cuisson d'argile et de calcaire à haute température, le ciment a tendance à être considéré comme une roche ignée artificielle. Sa réaction avec l'eau s'apparente à un processus de vieillissement accéléré qui aboutit à un mélange dense de minéraux hydratés que l'on appelle les hydrates de ciment - semblable à une roche sédimentaire. Les matériaux cimentaires durcis sont généralement des matériaux très peu perméables. Cependant, la perméabilité est une propriété essentielle car elle contrôle largement leur durabilité en milieu humide.

Très schématiquement, les questions soulevées par les matériaux peu perméables entrent dans deux catégories. Une catégorie regroupe les questions liées aux très petites dimensions des pores, tandis que l'autre est liée à la complexité et à la nature souvent hiérarchique ou multiéchelle de l'espace vide. Parmi les questions qui relèvent de la première catégorie, on peut citer l'influence de la dimension des pores, de la mouillabilité des parois des pores, de la courbure des parois des pores ou des frontières des pores. Cette physique n'exige pas l'étude de systèmes complexes. Elle peut être étudiée à l'aide de systèmes-modèles simples tels que les nanotubes, les tamis moléculaires ou le silicium poreux, en s'appuyant sur des expériences réelles ou une simulation moléculaire. Une question simple et pourtant très pertinente - en particulier pour les schistes ou les polymères - est la nature du transport moléculaire dans le cas extrême des matériaux subnanoporeux pour lesquels la dimension des pores est du même ordre que le diamètre moléculaire, c'est-à-dire des conditions dans lesquelles les concepts de base de l'hydrodynamique cessent de s'appliquer. Des études récentes ont montré que l'écoulement dans de telles conditions ressemble beaucoup à un glissement total avec frottement. Il s'apparente à la reptation, à savoir le type de mouvement de diffusion que l'on rencontre dans les polymères fondus, où chaque molécule de polymère glisse dans son « tube ». Fait intéressant, dans le cas d'un fluide unique, ceci ne remet pas en cause la relation linéaire entre le débit et le gradient de pression (ou de manière plus générale, entre le débit et le gradient de potentiel chimique). Il modifie toutefois le sens physique du coefficient de proportionnalité (la perméance), qui n'est plus le rapport entre la propriété d'un matériau (perméabilité) et la propriété d'un fluide (viscosité).

En fait, le glissement - éventuellement avec frottement - demeure une problématique importante dans un large éventail de conditions, y compris dans les écoulements électrocinétiques. En général, le nombre de Knudsen reste le principal paramètre à prendre en compte pour estimer l'éventuelle nécessité d'introduire des conditions aux limites de glissement, mais il semblerait que le traitement de Klinkenberg du glissement ne suffit plus. La valeur absolue de la dimension des pores et la courbure des parois des pores apparaissent comment des paramètres supplémentaires importants dont il faut tenir compte. Il reste à déterminer le lien que peuvent avoir ces derniers avec les phénomènes d'adsorption. 
Une autre classe de phénomènes qui mérite d'être étudiée de façon plus approfondie est celle des barrières contrôlées par les interfaces. Cela peut par exemple se produire lors du passage forcé de la phase liquide d'un hydrocarbure piégé dans un milieu hydrophobe nanoporeux (kérogène) vers un milieu hydrophile (fractures remplies d'eau). Dans ce cas, le taux de fuite n'est plus contrôlé par les propriétés de transport, mais par la barrière d'énergie interfaciale pour la nucléation et la formation de gouttelettes ou de bulles au niveau de l'interface hydrophobe-hydrophile.

Un aspect encore plus intrigant est la différence entre les propriétés de transport théoriques et mesurées (diffusivité ou perméabilité) dans les systèmes-modèles simples en milieux nanoporeux tels que les membranes de silicium zéolithique ou poreux. Les données expérimentales semblent être systématiquement très inférieures aux valeurs attendues en se basant sur les modèles conventionnels. Des barrières interfaciales à l'entrée des nanopores ont été évoquées, mais une explication physique de l'existence de ces barrières fait encore défaut. On peut soupçonner que la nature granulaire des molécules à ces échelles de longueur très petites soit impliquée, mais cela reste très hypothétique.

Pris ensemble, les divers phénomènes qui viennent d'être mentionnés - glissement, frottement, adsorption, mouillage, courbure, barrières interfaciales, etc., tous abordés au cours de la conférence LowPerm 2015 - représentent un formidable défi. Le fait qu'ils puissent faire collectivement l'objet d'un traitement commun reste à déterminer.

Une deuxième catégorie de questions bien différentes que soulève les milieux peu perméables sont celles liées à la nature complexe et souvent multiéchelle de l'espace vide. À l'ère du Big Data, beaucoup d'entre nous rêvent de la technique ultime qui permettrait de cartographier un Volume Élémentaire Représentatif (VER) avec une résolution atomique. Bien que cela puisse devenir une réalité dans un avenir pas trop lointain, cela n'est pas nécessairement une option souhaitable. Plus précisément, même une quantité astronomique d'informations structurelles ne nous apporterait pas tout ce que nous recherchons. Par exemple, même un concept simple comme la tortuosité n'est pas de nature purement géométrique. Elle dépend essentiellement d'une dynamique moléculaire interfaciale de courte durée. La réponse à des questions telles que « Combien de temps une molécule passe-elle sur la paroi d'une famille de pores donnée avant de revenir au cœur des pores ? » est tout aussi importante que la topologie de l'espace vide (connectivité) servant à estimer les facteurs de restriction du transport. Plutôt que de s'appuyer sur une technique unique et idéalement puissante pour obtenir les informations géométriques ultimes, il est probablement plus sage de faire appel à plusieurs techniques capables de fournir des informations spécifiques, que ce soit dans l'espace réel ou dans l'espace de Fourier. Parmi les différentes techniques que l'on peut envisager, la résonance magnétique nucléaire à bas champ mérite une mention spéciale. Elle donne accès à une très large gamme d'échelles de temps et de longueur et possède une capacité unique à corréler la dynamique dans différentes familles de pores.

Une situation particulièrement difficile est celle des milieux (micro)fracturés (sub)nanoporeux, où les (sub)nanopores sont les vides dans lesquels les molécules sont fortement adsorbées, tandis que les (micro)fractures sont les principales voies de fuite. Elle constitue probablement un bon modèle pour la production d'hydrocarbures à partir de schistes riches en matière organique. Il s'agit d'une situation difficile non seulement pour la caractérisation, mais également pour la modélisation. En raison des échelles de longueur et de la dynamique très différentes des deux types de porosité, il est peu probable que les modèles conventionnels à double porosité puissent être utilisés. En outre, une forte dépendance du réseau de (micro)fractures vis-à-vis de l'état de contrainte mécanique est attendue. La sensibilité à l'état de contrainte pourrait être encore plus élevée si les molécules en mouvement venaient à se dissoudre dans la matrice au lieu d'occuper les pores préexistants. Cette situation, combinée à des changements concomitants du volume de matières solides, se produit dans les barrières de polymères, dans les argiles gonflantes, et potentiellement dans le kérogène.

Une fois de plus, lorsqu'elles sont prises ensemble, les différentes sources d'hétérogénéité structurelle et/ou dynamique représentent un formidable défi pour adapter nos modèles à l'échelle macroscopique. Tout comme ce que nous avons pu observer au niveau local, il y a peu d'espoir que cela puisse se faire en présupposant un seul type d'écoulement (Darcy, diffusion, diffusion 
anomale, etc.) et en dissociant les propriétés du fluide (densité, mobilité, temps de séjour, etc.) de la structure solide. L'utilisation de méthodes de mise à l'échelle progressive basées sur les théories de la réponse linéaire d'Onsager et de Maxwell-Stefan, qui définissent la relation entre les flux et les gradients de potentiel chimique en intégrant un facteur de mobilité dépendant de la structure, éventuellement avec l'aide de méthodes de simulation moléculaire, pourrait se révéler être une approche plus efficace. 